\title{
Trillion Sensors and MEMS
}

\author{
Susumu Kaminaga ${ }^{*}$ \\ SPP Technologies Co., Ltd., 1-3-2 Otemachi, Chiyoda-ku, Tokyo 100-0004, Japan
}

(Received November 23, 2017; accepted January 29, 2018)

Keywords: Trillion Sensors (TSensors), microelectromechanical systems (MEMS), Internet of Things (IoT), deep reactive ion etching (DRIE), Bosch process, advanced silicon etch (ASE)

The Trillion Sensors (TSensors) Initiative was started in 2013 in the U.S. alongside the global economic tide of the Internet of Things (IoT) to pursue a society in which trillion sensors are connected to networks worldwide so that global problems may be solved in order to attain the utopian world, Abundance. It will provide all the people with food, medicine and healthcare, a clean environment, disaster resilience, and clean energy. TSensors, as a subset of the IoT that is becoming widely prevalent in recent years, have been enabled by microelectromechanical systems (MEMS), which have undergone dramatic growth in the last few decades and have been playing an essential role in the emerging world of IoT. One of the most essential technologies that has made possible the remarkable growth of MEMS is the deep reactive ion etching (DRIE) based on the Bosch process patented by Robert Bosch more than 20 years ago. The technology has been continuously developed to enhance its capability for over 20 years under the author's lead, in order to broaden the range of application of MEMS in automotives, gaming, smartphones, and sensor networks in the world of IoT.

\section{Introduction}

Under the circumstances that the Internet of Things (IoT) has become widely prevalent in recent years, the Trillion Sensors (TSensors) Initiative was started in 2013 in the U.S., aimed at realizing the TSensors Universe in ten years time in the middle of the 2020s. It is a society in which trillions of sensors are connected to networks yearly. Inspired by the conference held at Stanford University in 2010, Janusz Bryzek, vice president of Fairchild Semiconductor International at that time, initiated the concept of the TSensors Summit. His motivation was then reinforced by the book, Abundance ${ }^{(1)}$ in late 2012, in which a utopian world, Abundance, where food, medical care, a clean environment, and clean energy will be available to all people, is envisaged to be realized in 20 years. In this world, Abundance, everything will be covered with sensors connected to networks to solve the global problems. This will be realized thanks to eight exponential technologies: biotechnology, computers, networks and sensors, artificial intelligence, robotics, 3D printing, medicine, and nanotechnology. The networks and sensors is one of the exponential technologies as well as an enabler of the other technologies, and 45

*Corresponding author: e-mail: susumu.kaminaga@spp-technologies.co.jp

http://dx.doi.org/10.18494/SAM.2018.1814 
trillion sensors are predicted to be required by the late 2020s.

Such a massive movement as TSensors in the world of IoT has been enabled by microelectromechanical systems (MEMS) which has undergone remarkable growth in the last 20-25 years. In addition to MEMS sensors, wireless sensor networks have been applied to broader areas exploiting the unique features of MEMS and are playing an important role in the realization of the movement of TSensors in the world of IoT.

\section{TSensors}

The trillion sensors predicted to be used per year in the TSensors Universe are approximately 100 times the current usage and each of the current 7 billion people in the world would use approximately 140 sensors every year. All areas such as medicine/healthcare, agriculture, environment, and social infrastructure will be replete with sensors to provide connection to networks, and expanding the application of Big Data to broader areas will change the society and lifestyle drastically, leading to the solving of global problems, enabling the provision of food, distributed medicine/healthcare, a clean environment, and established clean energy for all the people. This describes the realization of Abundance in the coming 20 years (Fig. 1) in line with the emergence of the diversified global economic tide of IoT and Internet of Everything (IoE).

The genesis of the TSensors Initiative goes back to the MEMS Technology Summit Conference held at Stanford University in 2010. Following the visions of increasing the proliferation of sensors presented by experts at that conference, the exploration of sensors which were predicted to increase rapidly, was initiated by Janusz Bryzek. In accordance with his belief in the necessity of concentrated efforts to accelerate the development of sensors for commercialization toward TSensors usage, brainstorming by relevant people in the U.S. and Europe created new ideas and the concept of the TSensors Summit Conference was initiated by Bryzek. ${ }^{(2)}$ With the expectation that 45 trillion sensors will be required by the late $2020 \mathrm{~s}$, increasing from 1 billion and 10 billion in 2007 and 2014, respectively, the aim of the TSensors Initiative is to drastically shorten the development time of sensors so as to realize the world of Abundance. Hence, the objective of the TSensors Summit is to clarify, in the early stage,

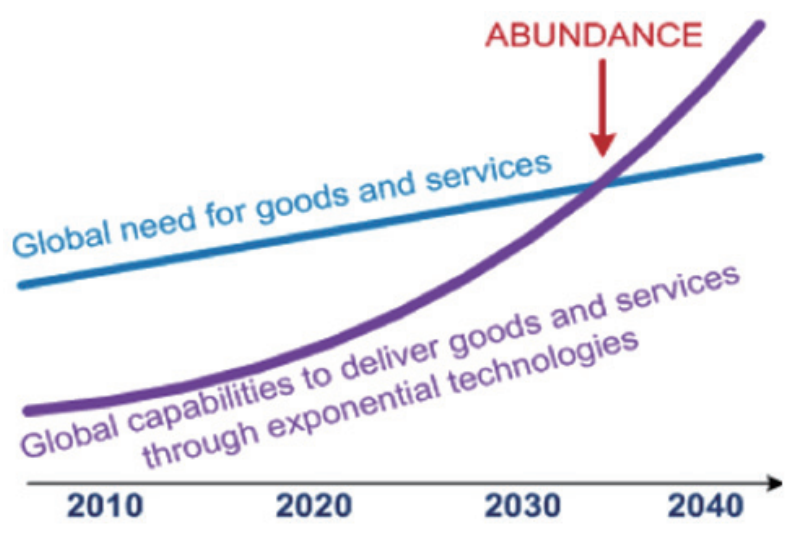

Fig. 1. (Color online) Exponential technologies promise growth of goods and services to match their global demand within one generation, enabling Abundance. ${ }^{(2)}$ 
the ultrahigh volume of sensor applications emerging in the future, referring to the prediction of experts from all over the world and to provide guidelines for the acceleration of sensor development under industry-academia-government collaboration. To make that real, the establishment of supporting technologies and supply chains is also targeted to accelerate the commercialization of sensors.

\section{TSensors Summits}

Following the TSensors Universe Workshop held at the University of California Berkeley in March 2013, the first TSensors Summit was inaugurated at Stanford University in October 2013. Further summits were held in Tokyo in February 2014, ${ }^{(3)}$ Munich in September 2014, San Diego in November 2014, and Tokyo again in December 2014. Exciting discussion was conducted at every summit with 150-300 participants. At the first TSensors Summit at Stanford University, many eminent players from IT, semiconductor and electronics industries, universities and research institutes, and well-known MEMS leaders, mainly from the U.S. and Europe, gathered together. More than 50 experts, including the author as the only Japanese speaker, were invited from all over the world to give speeches. Discussion was carried out with 250 participants in total. Approximately 300 applications were presented at the summit, classified into various sectors such as healthcare, environment, infrastructure, food production, energy, and automotives. The supporting technologies such as 3D printing, energy harvesting, wireless communication with ultralow power consumption, and security were also presented for the realization of TSensors. The following TSensors Summits provided additional ideas and a working group for the selection of applications to be focused on was planned to maximize global economic effects.

The creation of an amazing 18 trillion U.S. dollars business opportunity is expected in the 2020s. While drastic restructuring of the global workforce is anticipated with $60 \%$ of the current one expected to be lost, the creation of high skill jobs is required. It is suggested that large-scale education worldwide will be necessary to avoid serious social unrest. The TSensors Summit held in Florida, U.S., in December 2015 focused on the four pillars, namely, plentiful food, medical care, clean environment, and clean energy for all (Fig. 2), and also, printed electronics/sensors, as one of the most promising technologies to support TSensors, was discussed.

\section{TSensors Universe}

In 2025 , the total population on this planet will be 8 billion, of which 1.2 billion will be over the age of 60 . In the case of Japan, $30 \%$ of the total population will be over 60 years old in 2025. Japan has been facing the problems of an aging society, aging infrastructure, disaster prevention/mitigation, and the preservation of a sustainable environment as a front runner in the world, which is a position with obligations to strive to reach a solution.

The need to establish a comfortable life for elderly people with a safe and secure living atmosphere is an essential issue for the future, and preventive and predictive medicine are crucial from the viewpoint of reducing the seriously increasing medical cost. Unobtrusive 


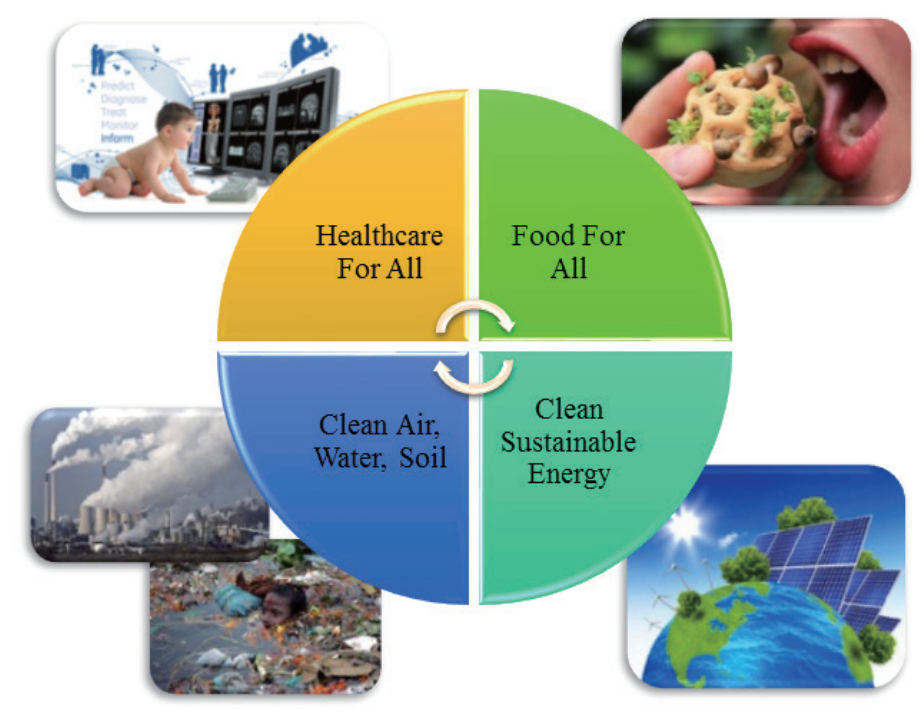

Fig. 2. (Color online) Trillion Sensors focused on these challenges. ${ }^{(4)}$

health monitoring sensors embedded in our connected surroundings of daily life will become essential in the future. For example, wearable sensors embedded in finger rings, wristbands, glasses, and clothes will be used in healthcare applications. Adhesive plaster health sensors and portable blood/glucose sensors will be used for non- and minimally invasive healthcare monitoring. Moreover, various kinds of services for aged people will be in place and the wireless sensor network (Fig. 3) will enable a 24-h healthcare service that provides throughthe-day at-home remote medical diagnosis. An elderly person monitoring system, for example, is expected to be developed with sensor monitoring systems in a close relationship with local societies. Care-assistance robots, drones, working robots, and autonomously driving cars will greatly expand the application of MEMS sensors.

Another requirement of a comfortable life is ensured safety, security, and cleanness in the social infrastructure and environment. Sensor monitoring of social infrastructure and the environment is needed for preventive and predictive maintenance of aged bridges, roads, tunnels, and sites of possible landslides to prevent or mitigate disaster. Monitoring with sensors will be implemented for the prevention of pollution such as radiation, particulate materials, and residual chemicals in the air and in drinking/waste water in the living environment. In the case of buildings, sensors will be installed in the structures to detect vibration and acceleration. This will give added value and ensure safe living conditions for the inhabitants. The sensors to be applied for such purposes include accelerometers, strain meters, inclinometers, optical fibers, imaging sensors, and acoustic emission (AE) sensors. Inside houses and buildings, sensors will monitor temperature, humidity, illumination level, oxygen, carbon dioxide and gas concentrations, and presence of fire so that inhabitants can feel comfortable. Many more emerging applications based on the wireless sensor network system will be feasible in the TSensors Universe (Fig. 4). 


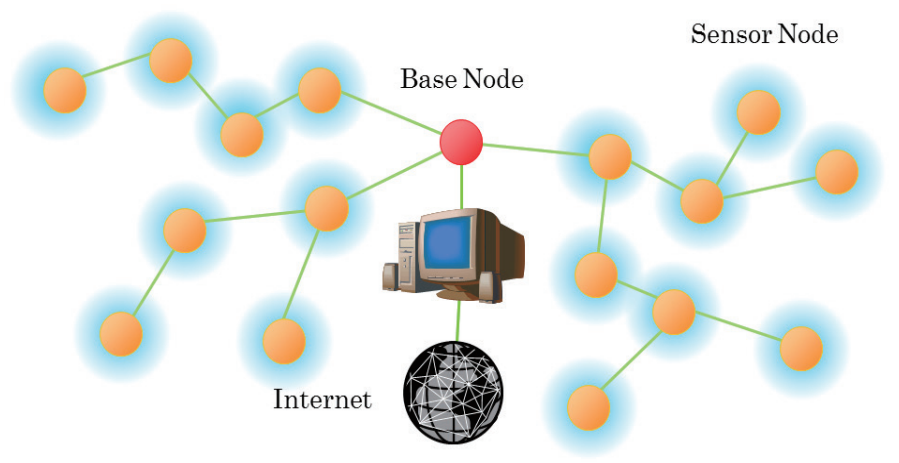

Fig. 3. (Color online) Wireless sensor network.

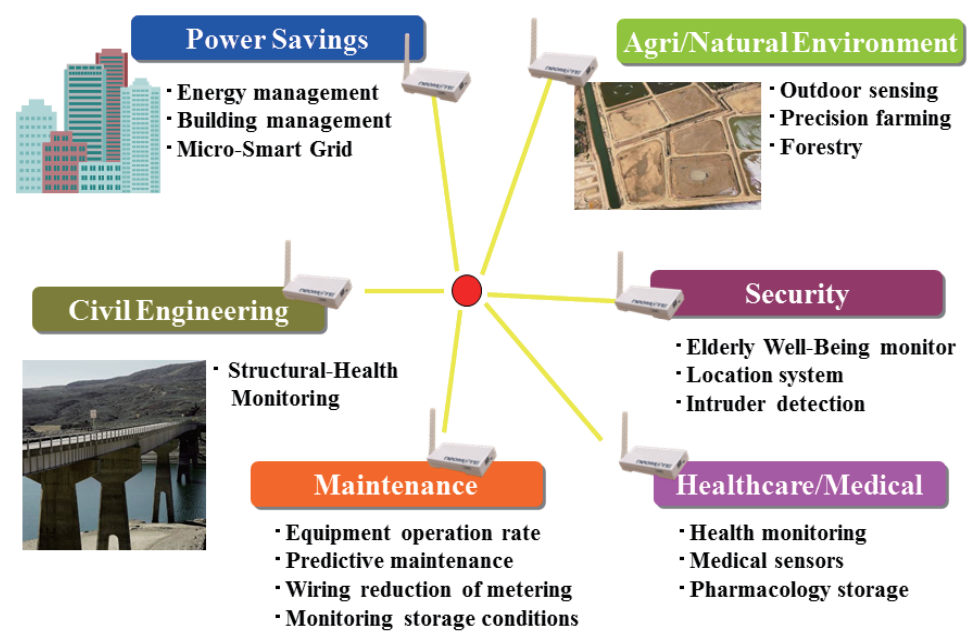

Fig. 4. (Color online) Applications of wireless sensor network.

\section{History of MEMS}

As described above, MEMS sensors have been playing a key role toward realizing IoT and TSensors these last few decades. Historically, research work and the commercialization of MEMS were already accomplished by Masayoshi Esashi at Tohoku University in the early 1970s. ${ }^{(5)}$ In 1982, Kurt E. Petersen of IBM Research Laboratory, U.S., wrote a paper, "Silicon as a Mechanical Material", ${ }^{(6)}$ in which he suggested that mechanical components can be produced with semiconductor processing technology. The term "MEMS" was coined in the U.S. in 1987.

The author was inspired by the MEMS movement in the U.S. in the late 1980s and, while aiming for the development of MEMS devices including sensors, started on the development and commercialization of the microprocessing technology. At Surface Technology Systems (STS), U.K., a subsidiary of Sumitomo Precision Products (SPP), Japan, at that time, currently SPTS Technologies, deep reactive ion etching (DRIE) was developed on the basis of the "Bosch process" ${ }^{\text {(7) }}$ patented by Robert Bosch, and the world's first DRIE equipment was introduced into the market in 1995 under the initiative of the author dispatched to the subsidiary from SPP. Since then, MEMS have undergone rapid growth thanks to the DRIE technology that has been 
enhanced with improved performance to satisfy the new requirements of customers who aim to develop new MEMS devices. MEMS have been applied to, for example, automotives, inkjet printers, displays, gaming, and smartphones with accelerometers, gyrosensors, and microphones incorporated.

Furthermore, wireless sensor networks utilizing the superior characteristics of MEMS sensors are used in various fields and applied in energy saving, alternative energy, social infrastructure, environment protection, safe and secure society, and healthcare.

\section{MEMS Processing Technologies}

While MEMS processing technologies originated from semiconductor processing technologies consisting of oxidation, doping, photolithography, etching, deposition, and evaporation, there are unique MEMS processing technologies, represented by silicon DRIE, sacrificial layer etching, and wafer-to-wafer bonding, that are not required for conventional semiconductor processing. Those technologies enable sensors, mechanical switches, and actuators to be processed on silicon substrates with the thicker 3D structures necessary for mechanical motion. This results in the necessity of a higher etching rate, higher selectivity to the mask, and highly precise processing.

Above all, the DRIE technology has been recognized as a revolutionary technology that has enabled the tremendous growth of MEMS in the last few decades. ${ }^{(8)}$ Beyond silicon, the microprocessing technologies have been developed also for other materials such as silicon carbide ( $\mathrm{SiC})$, gallium nitride $(\mathrm{GaN})$, and aluminum nitride (AlN).

\section{DRIE}

The cyclic etch/passivation method for anisotropically etched silicon, that is, the Bosch process, provides high etch rates, anisotropic profiles, and high selectivity to conventional photoresist masks. An outline of the process is shown in Fig. 5. First, in the passivation step, carbon fluoride (CF) polymerized film is deposited on the whole surface including the mask [Fig. 5(a)]. In the second step, while the film at the bottom of the profile is removed by ion bombardment, pulled by the bias voltage applied to the substrate, the film on the sidewall is not removed and remains because of the directional ions [Fig. 5(b)]. Then, only the exposed silicon at the bottom is etched by fluorine radicals [Fig. 5(c)]. This sequential process is repeated and anisotropic etching for obtaining a high-aspect-ratio profile is achieved, as shown in Figs. 5 and 6. More developed features were added to the original Bosch process at STS to enhance the process and this new technology was called the advanced silicon etch (ASE). ${ }^{(9,10)}$ It has greater reliability, applicability, and versatility and led to the world's first DRIE equipment.

As the plasma source that suits the Bosch process, inductively coupled plasma (ICP) is adopted to ensure a higher etch rate that provides higher resolution and density as well as better controllability of passivation with the capability to provide low to high resolution. The equipment comprises a plasma-generating chamber with dielectric on the outer surface and an exterior antenna coil. Plasma is generated with a high-frequency magnetic field induced inside 


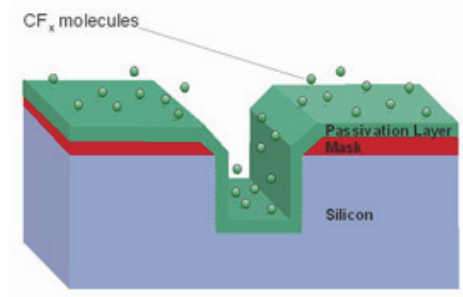

(a)

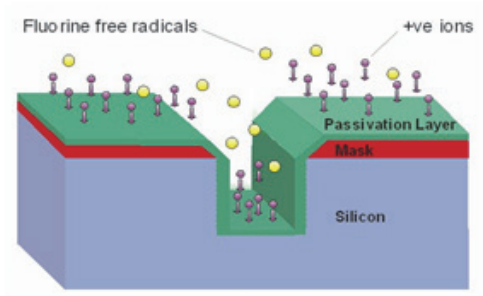

(b)

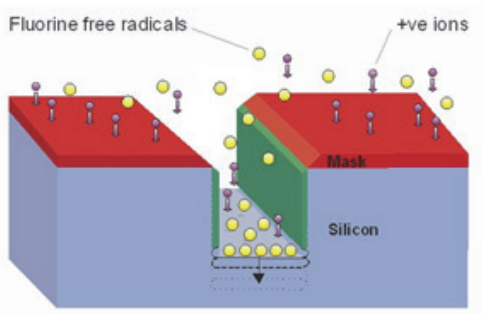

(c)

Back to step(a)

Fig. 5. (Color online) Bosch process. (a) Passivation step, (b) passivation removal step, and (c) etching step.
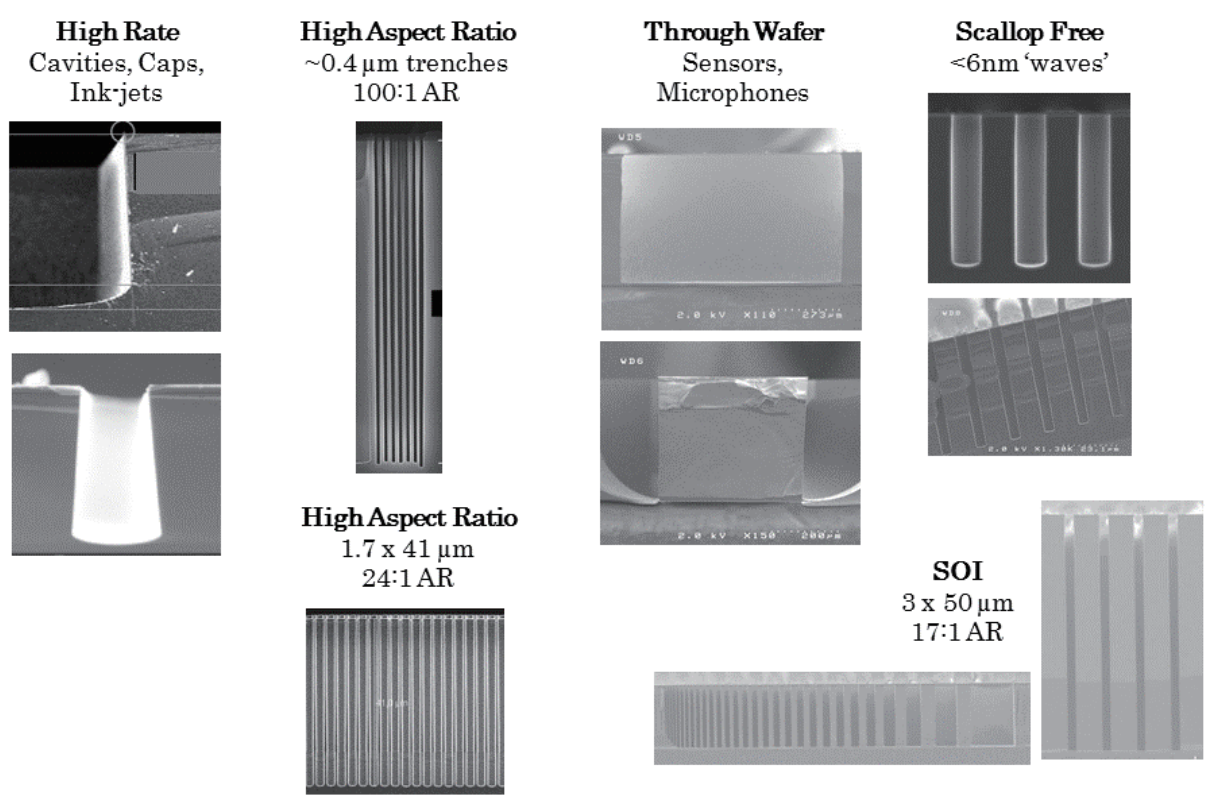

Fig. 6. Leading DRIE capability.

by applying high-frequency power to the antenna. With this relatively simple configuration, a high-density plasma can be generated. The method of supporting the substrate is to use an electrostatic chuck and mechanical clamping. High-frequency power is applied to the lower electrode in addition to the higher one to provide bias potential. ASE-Pegasus was developed for large-scale production with $200 \mathrm{~mm}$ wafers to achieve both higher etch rate and withinwafer uniformity with a superior profile, benefited by the originally designed ICP source. It enables highly dense, uniform plasma to be generated, and the chamber design supports efficient supply of the produced fluorine radicals to the wafers. As the performance of MEMS highly depends on the processed profile, and, in particular, the elimination of tilting of the profile within the wafer, control of the etched angle to obtain high uniformity within the wafer is required to achieve high-yield production of high-performance MEMS sensors. Tilting is caused mainly by the non-uniformity of plasma density and bias voltage applied to the edge of the wafer. 
To cope with the above challenges, ASE-Predeus has been developed to achieve an ultrahighuniformity plasma source with an enhanced design on top of ASE-Pegasus, as well as ultimate uniformity of the bias voltage at the wafer edges.

Moreover, ASE-Proxion has been developed to achieve both a high etch rate and high uniformity. It was difficult to achieve those two features for the larger exposed-area patterning necessary for microphones and cavity structures. That was because a larger number of fluorine radicals are used, and thus the excess etchant outside the wafer results in a higher etch rate at the wafer edge region. With the same uniformity as ASE-Pegasus, ASE-Proxion achieved a $100 \%$ higher etch rate. Also, instead of the conventional CF, ASE-Proxion employs an alternative deposition gas that has a lower global warming potential. In addition to the effect of benefiting environmental protection, the new gas provides shorter passivation time resulting in increased throughput. The continuous evolution of DRIE technology is shown in Fig. 7.

\section{Open Collaboration}

The TSensors Initiative suggests that sensors connected to networks play essential roles in every area in the TSensors Universe. However, it is not simple sensor development but a creation of new applications to solve global problems. One of the most essential aims of the TSensors Universe is to establish effective collaborative working systems and create new business models to implement data obtained with sensors into the actual society. From that point of view, the way to proceed from research and development to commercialization and further, industrialization, must be seriously considered. Successful commercialization can be achieved by fusing the different technologies developed through cross-functional collaboration of researchers and engineers as well as collaborative work of different specialists under joint

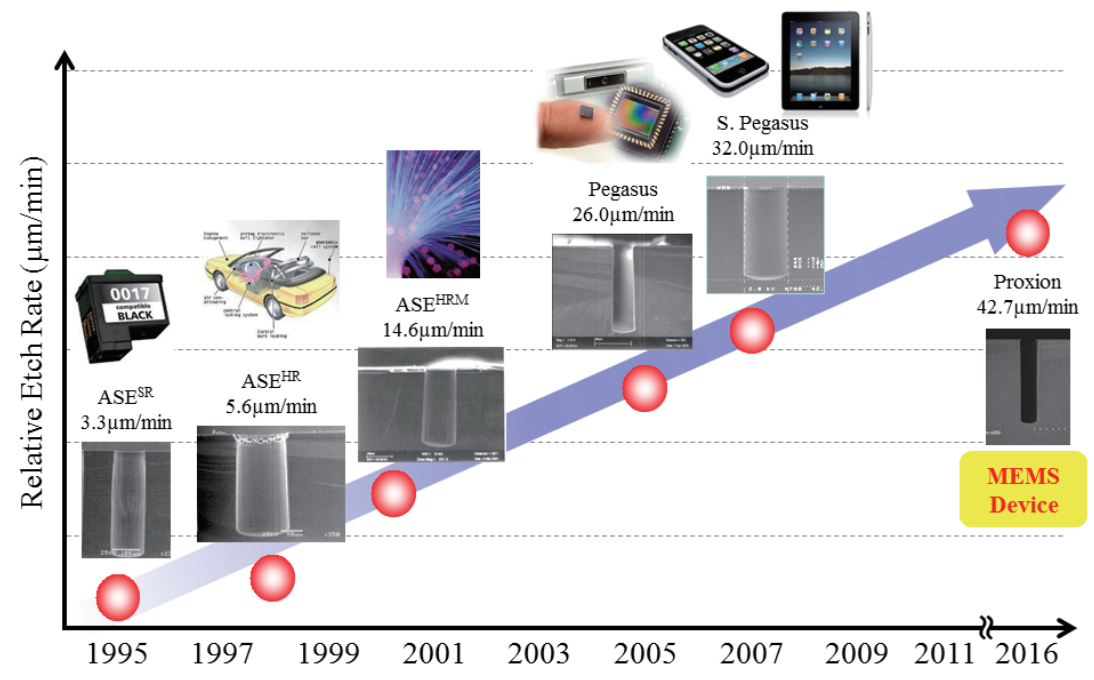

Fig. 7. (Color online) Continuous evolution of DRIE technology. 
work of academia and industry. Breaking silos in any organization will result in a common platform being established, where researchers, engineers, managers, and other relevant specialists can contribute to collaborative work to pursue the goals.

\section{Conclusion}

The objective of the TSensors Initiative is to realize the utopian world, Abundance. MEMS and sensors play an essential role in making it come true. DRIE technology has enabled MEMS and sensors to undergo dramatic growth in the past few decades, and the performance is being continuously enhanced to support the further development of MEMS and sensors. While emerging MEMS and sensors are expected to contribute to TSensors, new applications are to be created utilizing the data obtained by the sensors, in order to solve global problems. For that purpose, collaborative work among experts in relevant fields is essential from the viewpoint of a successful progression from research and development to commercialization and industrialization.

\section{References}

1 P. H. Diamandis and S. Kotler: Abundance (Free Press, 2012).

2 J. Bryzek: TSensors Summit for Trillion Sensor Roadmap (2013) 151.

3 J. Bryzek: Trillion Sensors Summit Japan 2014 (2014).

4 S. Whalley: American Physical Society, Actualization of the Internet of Things (April 17, 2017).

5 M. Esashi and T. Matsuo: Supplement to J. Jpn. Soc. Appl. Phys. 44 (1975) 339.

6 K. E. Petersen: Proc. IEEE 70 (1982) 420.

7 F. Laermer and A. Schilp: Method for Anisotropically Etching Silicon, German patent DE4241045 (1994) and U.S. patent 5501893 (1996).

8 S. Kaminaga: First Int. Symp. Integrated Microsystems (2011) 175.

9 J. Bhardwaj and H. Ashraf: Proc. SPIE Micromachining and Microfabrication Symp., SPIE-2639 (1995) 224.

10 J. Bhardwaj, H. Ashraf, and A. McQuarrie: Symp. Microstructures and Microfabricated Systems at the Annual Meeting of the Electrochemical Society (1997). 